\title{
OPEN Clinical consequences of head and neck free-flap reconstructions in the DM population
}

\author{
Ting-Han Chiu ${ }^{1}$, Chung-Kan Tsao ${ }^{2}$, Sheng-Nan Chang ${ }^{3,5 \bowtie}$, Jou-Wei Lin ${ }^{3} \&$ \\ Juey-Jen Hwang ${ }^{3,4,5 凶}$
}

Diabetes mellitus (DM) is a common comorbidity and risk factor for postoperative complications in head and neck ( $\mathrm{H} \& \mathrm{~N})$ microsurgical reconstructions. Our study focused on the association between DM and individual complications regarding both surgical and medical aspects. A meta-analysis of English-language articles comparing a series of complications between DM and non-DM H\&N free-flap recipients was performed by comprehensive meta-analysis (CMA). Twenty-seven articles presented $14,233 \mathrm{H} \& \mathrm{~N}$ free-flap reconstructions, and a subset of 2329 analyses including diabetic cases was included for final analysis. Total postoperative $(\mathrm{RR}=1.194, p<0.001 ; \mathrm{OR}=1.506, p=0.030)$ and surgical $(R R=1.550, p=0.001 ; O R=3.362, p<0.001)$ complications were increased in DM subjects. Free-flap failure/necrosis ( $R R=1.577, p=0.001 ; O R=1.999, p=0.001$ ) and surgical site infections $(O R=2.414, p<0.001)$ were also increased in diabetic recipients. However, return to the operating room, dehiscence, fistulas, plate exposures, readmissions, and mortalities were not increased in DM patients. DM increased various complications in H\&N free-flap reconstructions. Surgical indications should be cautiously evaluated, and aggressive treatments should be implemented for high-risk recipients.

Free-flap reconstruction following head and neck $(\mathrm{H} \& \mathrm{~N})$ tumor ablation has become a routine practice with advancements in microsurgical techniques ${ }^{1}$. However, daunting postoperative complications after free-flap reconstruction, such as deprivation of eating, speaking, and breathing functions, have been reported $^{2}$. Numerous practices have been introduced to improve surgical outcomes after free-flap reconstruction, especially risk factor evaluation ${ }^{3}$. Among those factors associated with postoperative complications, diabetes mellitus (DM) is a very important risk factor in clinical practice. However, there have been differing reports about free-flap success rates, surgical site infections, and surgical outcomes in DM patients in previous studies ${ }^{4-7}$.

A lack of persuasive and clinical evidence impedes determining whether DM is contributory to complications following major microsurgical procedures. As tissues left after $\mathrm{H} \& \mathrm{~N}$ tumor resections are often scarce, free-flap reconstruction is frequently used for instant coverage and functional restorations. Serious postoperative complications after free-flap reconstruction not only psychologically devastate patients with physical appearance or loss of basic function but also may delay crucial radiotherapy ${ }^{4,5}$. In those cases, the quality of life after the operation is not satisfactory due to repeated readmissions or revision operations ${ }^{8-10}$. Therefore, recognizing the risk of DM in association with free-flap reconstruction and the response to corresponding treatments before free-flap reconstruction are very important in real-world practice. To define the possible effects of DM in association with free-flap use for $\mathrm{H} \& \mathrm{~N}$ reconstruction, we performed this meta-analysis to examine these associated complications in more detail.

\footnotetext{
${ }^{1}$ Department of Surgery, Chang Gung Memorial Hospital and Chang Gung University College of Medicine, Taoyuan, Taiwan. ${ }^{2}$ Department of Plastic and Reconstructive Surgery, Chang Gung Memorial Hospital and Chang Gung University College of Medicine, Taoyuan, Taiwan. ${ }^{3}$ Division of Cardiology, Department of Internal Medicine, National Taiwan University Hospital Yun-Lin Branch, Dou-Liu City, Taiwan. ${ }^{4}$ Division of Cardiology, Department of Internal Medicine, National Taiwan University Hospital, Taipei, Taiwan. ${ }^{5}$ These authors contributed equally: Sheng-Nan Chang and Juey-Jen Hwang. ${ }^{\circledR}$ email: p95421008@ntu.edu.tw; jeremysnc1000@gmail.com
} 


\section{Patients and methods}

Inclusion criteria. All published original studies of either randomized control trials or retrospective cohorts mentioning DM and any certain complications following H\&N free-flap reconstructions were included. Articles by the same authors or based on the same study population should be verified as separate studies or designated to analyze a different complication before being included.

Search strategy. A search of electronic databases, namely PubMed Central, Embase, MEDLINE, and the Cochrane Library, from January 2005 to April 2020 was conducted; the search terms were "DM", "H\&N," AND "free flaps." This was further supplemented with cross-referencing the bibliographies from the papers identified by the search or other relevant articles. Only full-text manuscripts in the English language were considered for inclusion. For studies with overlapping periods of the same targeted population, the one with the largest cohort was retained, while additional consideration for inclusion was made if the overlapping paper mentioned a specific complication that was not previously covered. Articles without available documentation of the diabetic or nondiabetic case numbers and respective complication rates or without an odds ratio comparing risks in diabetic to those in nondiabetic patients were further excluded.

Data extraction. After selecting the relevant literature, the primary data collection was performed and further reviewed by a second author to ensure accuracy. The data collected were as follows: definition of the reconstructed regions, study population and years, numbers of total patients and patients with DM, and rates of complications in both patients with and without DM. Various complications (surgical and medical), surgical complications, return to the operating room, free-flap failure/necrosis, surgical site infections, dehiscence, hematomas, coagulation-related complications (e.g., hematoma, bleeding, requiring transfusion or thrombosis), fistulas, plate exposures, other complications, readmissions, and mortality were included in the complication analysis.

Statistical analysis. The meta-analysis was conducted using Comprehensive Meta-Analysis (CMA) 2.0 Software, [Biostat, New Jersey, USA], using the inverse variance method for pooled relative risk. The dichotomous data were summarized using relative risk (RR) or odds ratio (OR) separately and with $95 \%$ confidence intervals (95\% CIs). Statistical heterogeneity tests of Cochran's Q-value, $I^{2}$, and $\mathrm{Tau}^{2}$ were performed to determine whether a fixed or random effect model was adopted.

\section{Results}

Process outcomes. The search strategy identified 63 eligible studies, and 27 studies were ultimately included in the meta-analysis ${ }^{5,11-37}$. The selection process is demonstrated in the flow diagram (Fig. 1), and the study characteristics are presented in Table 1. The funnel plot of all studies included is presented in Fig. 2.

Analysis of complications. Total complications. A total of 6 studies were included for overall postoperative, surgical and other complications. A total of 3924 patients were enrolled, 466 of whom had DM. The pooled results were 6 studies with RR and 3 studies with OR. After multivariable analysis, DM significantly increased the total complication rate $(\mathrm{RR}=1.194,95 \% \mathrm{CI} 1.089-1.310, p<0.001$ and $\mathrm{OR}=1.506,95 \%$ CI $1.040-2.181$, $p=0.030)$ (Fig. 3).

Total and individual surgical complications. Total surgical complications. Ten studies that evaluated total surgical complications were identified; a total of 5064 patients were enrolled, 744 of whom were DM patients. The pooled results include 9 studies with RR and 3 studies with OR. The multivariate analysis results showed that DM significantly increased surgical complication rates $(\mathrm{RR}=1.550,95 \% \mathrm{CI} 1.195-2.010, p=0.001$ and $\mathrm{OR}=3.362$, 95\% CI 1.961-5.763, $p<0.001$ ) (Fig. 3).

Return to the operating room. Five studies evaluating the rates of "return to the operating room" were included, with 413 DM patients among 2976 total subjects. All complication rates were expressed as RR. The pooled results showed that DM did not significantly increase the rate of "return to the operating room" $(\mathrm{RR}=1.415,95 \% \mathrm{CI}$ $0.760-2.633, p=0.273$ ) (Fig. 4).

Flap failure/necrosis. Ten studies that evaluated flap failure or necrosis were included, with 9144 total patients and $1550 \mathrm{DM}$ patients. The pooled results included 9 studies with RR and 3 studies with OR. The multivariate analysis results showed that DM significantly increased flap failure or necrosis ( $R R=1.577,95 \%$ CI $1.217-2.043$, $p=0.001$ and $\mathrm{OR}=1.999,95 \%$ CI $1.347-2.967, p=0.001$ ) (Fig. 4).

Surgical site infections. A total of 11 studies evaluated surgical site infections, with 5488 patients enrolled; 773 of them had DM. The pooled results included 8 studies with RR. There was a trend of increasing surgical site infections in the DM group, nearly reaching statistical significance $(\mathrm{RR}=1.401,95 \% \mathrm{CI} 0.987-1.987, p=0.059)$. After pooling the results from the 5 studies that reported OR, DM significantly increased surgical site infections $(\mathrm{OR}=2.414,95 \%$ CI 1.619-3.599, $p<0.001)$ (Fig. 4). 
Studies identified through PubMed,

Embase, MEDLINE, Cochrane Library ( $n=1243)$

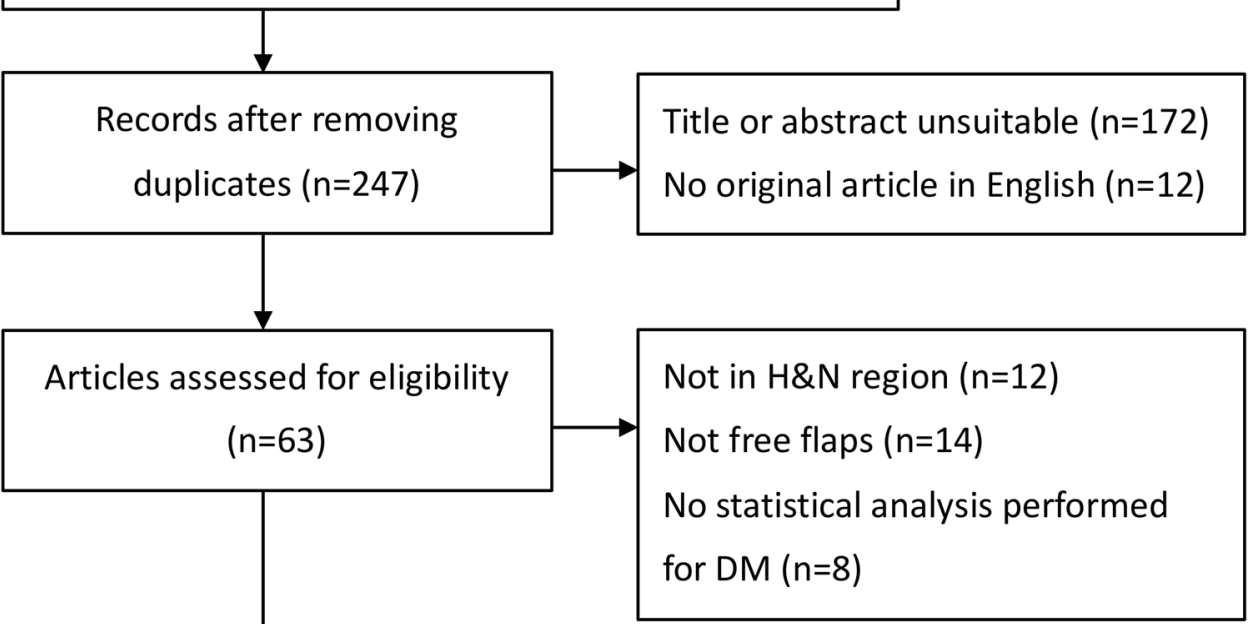

Figure 1. The flow diagram shows the process of enrolling studies for the meta-analysis.

Dehiscence. Four studies were included to evaluate dehiscence rates, with $473 \mathrm{DM}$ patients among 3311 total subjects. All complication rates were expressed as RR. The pooled results showed that DM did not significantly increase dehiscence rates $(\mathrm{RR}=1.162,95 \%$ CI 0.814-1.660, $p=0.408)$ (Fig. 5).

Fistula. Two studies were included to evaluate fistula formation after surgery, with 63 DM patients among 723 subjects. All complication rates were expressed as RR. The pooled results showed that DM did not significantly increase fistula formation after the operation $(\mathrm{RR}=4.323,95 \% \mathrm{CI} 0.172-108.961, p=0.374)$ (Fig. 5).

Plate exposure. Two studies were included for the evaluation of plate exposures, with $67 \mathrm{DM}$ patients among 665 subjects. All complication rates were expressed as RR. The pooled results showed that DM did not increase plate exposure rates $(\mathrm{RR}=0.944,95 \%$ CI $0.374-2.387, p=0.904)$ (Fig. 5).

Hematoma. Four studies were included for the evaluation of hematoma, with 176 DM patients among 1150 subjects. All complication rates were expressed as RR. The pooled results showed that DM did not significantly increase the rates of hematoma $(\mathrm{RR}=3.338,95 \% \mathrm{CI} 0.742-15.025, p=0.116)$ (Fig. 5).

Coagulation-related complications. Regarding coagulation-related complications (e.g., hematoma, bleeding, required transfusion, and thrombosis), seven studies were included in the meta-analysis. The complication rates were expressed as RR; there were a total of 3849 patients, 562 of whom were DM patients. The pooled results showed that there was a trend of increasing coagulation-related complications in the DM group $(\mathrm{RR}=1.708$, 95\% CI 0.918-3.178, $p=0.091$ ) (Fig. 5).

Quality aspects. Readmissions. Two studies were included to evaluate the rates of readmission, with 340 DM patients among 2702 subjects. All complication rates were expressed as RR. The pooled results showed that DM did not significantly increase readmission rates $(\mathrm{RR}=1.211,95 \% \mathrm{CI} 0.870-1.686, p=0.256)$ (Fig. 6). 


\begin{tabular}{|c|c|c|c|c|c|c|}
\hline References & Study Design & Database & Flap definition & No. of patients & No. of DM patients & Analyzed complication \\
\hline Bozikiv $^{11}$ & Retrospective cohort & $\begin{array}{l}\text { University Medical Centre } \\
\text { Ljubljana, Slovenia } \\
\text { 1989-1999 }\end{array}$ & $\begin{array}{l}\text { H\&N free flaps following } \\
\text { tumor ablations }\end{array}$ & 162 & 12 & $\begin{array}{l}\text { Surgical complications (RR), } \\
\text { flap failure/necrosis (RR) }\end{array}$ \\
\hline Valentini ${ }^{12}$ & Retrospective cohort & $\begin{array}{l}\text { Polyclinic "Umberto I," Uni- } \\
\text { versity of Rome, Italy } \\
\text { 2001-2004 }\end{array}$ & $\mathrm{H} \& \mathrm{~N}$ free flaps & 118 & 8 & $\begin{array}{l}\text { Return to OR (RR), hema- } \\
\text { toma (RR) }\end{array}$ \\
\hline Naura $^{13}$ & Retrospective cohort & $\begin{array}{l}\text { Cleveland Clinic, United } \\
\text { States } \\
6 \text { years }\end{array}$ & $H \& N$ free flaps & 300 & 28 & $\begin{array}{l}\text { Surgical complications } \\
\text { (RR), return to OR (RR), } \\
\text { flap failure/necrosis (RR), } \\
\text { surgical site infection (RR), } \\
\text { dehiscence (RR), hematoma } \\
\text { (RR), fistula (RR), plate } \\
\text { exposure (RR) }\end{array}$ \\
\hline $\mathrm{Kao}^{14}$ & Retrospective cohort & $\begin{array}{l}\text { Chang Gung Memorial } \\
\text { Hospital, Taiwan } \\
\text { 2000-2008 }\end{array}$ & $\begin{array}{l}\text { H\&N free flaps after cancer } \\
\text { ablations }\end{array}$ & 62 & 16 & $\begin{array}{l}\text { Surgical complications (RR), } \\
\text { return to OR (RR) }\end{array}$ \\
\hline Bianchi $^{15}$ & Retrospective cohort & $\begin{array}{l}\text { University of Parma, Italy } \\
2000-2007\end{array}$ & $H \& N$ free flaps & 352 & 24 & Total complications (RR, OR) \\
\hline $\mathrm{Joo}^{16}$ & Retrospective cohort & $\begin{array}{l}\text { Catholic University of Korea, } \\
\text { Korea } \\
1993-2009\end{array}$ & $\begin{array}{l}\text { H\&N free flaps after ablative } \\
\text { surgery }\end{array}$ & 237 & 27 & $\begin{array}{l}\text { Flap failure/necrosis (RR, } \\
\text { OR) }\end{array}$ \\
\hline $\operatorname{Lee}^{17}$ & Retrospective cohort & $\begin{array}{l}\text { University of Jena, Germany } \\
2008-2009\end{array}$ & $\mathrm{H} \& \mathrm{~N}$ free flaps & 81 & 7 & $\begin{array}{l}\text { Surgical complications (RR, } \\
\text { OR) }\end{array}$ \\
\hline le Nobel ${ }^{18}$ & Retrospective cohort & $\begin{array}{l}\text { Sunnybrook Health Science } \\
\text { Center, Canada } \\
\text { 2003-2010 }\end{array}$ & $\mathrm{H} \& \mathrm{~N}$ free flaps & 289 & 35 & Total complications (RR, OR) \\
\hline Vandersteen $^{19}$ & Retrospective cohort & $\begin{array}{l}\text { Institut Universitaire de la } \\
\text { Face et du Cou, France } \\
2000-2010\end{array}$ & $\mathrm{H} \& \mathrm{~N}$ free flaps & 423 & 35 & $\begin{array}{l}\text { Total complications (RR), } \\
\text { surgical complications (RR), } \\
\text { surgical site infection (RR), } \\
\text { hematoma (RR), fistula (RR), } \\
\text { mortality (RR) }\end{array}$ \\
\hline Riva $^{20}$ & Retrospective cohort & $\begin{array}{l}\text { Kaohsiung Chang Gung } \\
\text { Memorial Hospital, Taiwan } \\
\text { 1996-2008 }\end{array}$ & $\begin{array}{l}\text { H\&N free flaps after abla- } \\
\text { tions }\end{array}$ & 1233 & 189 & Flap failure/necrosis (RR) \\
\hline Avery $^{21}$ & Retrospective cohort & $\begin{array}{l}\text { University Hospitals of } \\
\text { Leicester, United Kingdom } \\
\text { 1996-2012 }\end{array}$ & $\begin{array}{l}\text { Free pectoralis major flaps } \\
\text { for maxillofacial regions }\end{array}$ & 100 & 16 & Flap failure/necrosis (OR) \\
\hline $\mathrm{Liu}^{22}$ & Retrospective cohort & $\begin{array}{l}\text { Shanghai Ninth People's } \\
\text { Hospital, China } \\
\text { 2003-2013 }\end{array}$ & $\begin{array}{l}\mathrm{H} \& \mathrm{~N} \text { free flaps for oral } \\
\text { cancers }\end{array}$ & 309 & 105 & $\begin{array}{l}\text { Surgical complications (RR, } \\
\text { OR), return to OR (RR), } \\
\text { flap failure/necrosis (RR), } \\
\text { surgical site infection (RR), } \\
\text { dehiscence (RR), hematoma } \\
\text { (RR), thrombosis (RR) }\end{array}$ \\
\hline Mitchell $^{23}$ & Retrospective cohort & \begin{tabular}{|l} 
University of Washington \\
Medical Center or Harbor- \\
view Medical Center, Unites \\
States \\
$2006-2013$
\end{tabular} & $\begin{array}{l}\text { H\&N free flaps (clean-con- } \\
\text { taminated wounds) }\end{array}$ & 427 & 40 & Surgical complications (OR) \\
\hline $\mathrm{Lo}^{24}$ & Retrospective cohort & $\begin{array}{l}\text { Cathay General Hospital, } \\
\text { Taiwan } \\
\text { 2010-2014 }\end{array}$ & $\begin{array}{l}\text { H\&N free flaps after cancer } \\
\text { ablations }\end{array}$ & 158 & 32 & Total complications (RR) \\
\hline Ishimaru $^{25}$ & Retrospective cohort & $\begin{array}{l}\text { National Inpatient Database, } \\
\text { Japan } \\
2010-2013\end{array}$ & $\begin{array}{l}\mathrm{H} \& \mathrm{~N} \text { free flaps after tumor } \\
\text { resections }\end{array}$ & 2846 & 737 & $\begin{array}{l}\text { Flap failure/necrosis (RR, } \\
\text { OR) }\end{array}$ \\
\hline Eder-Czembirek $^{26}$ & Retrospective cohort & \begin{tabular}{|l} 
Vienna General Hospital, \\
Austria \\
$2004-2011$
\end{tabular} & $\begin{array}{l}\text { Free-flap reconstructions } \\
\text { for oral squamous cell } \\
\text { carcinoma }\end{array}$ & 85 & 9 & $\begin{array}{l}\text { Surgical site infection (RR, } \\
\text { OR) }\end{array}$ \\
\hline Zhou $^{27}$ & Retrospective cohort & $\begin{array}{l}\text { Peking University School } \\
\text { and Hospital of Stomatology, } \\
\text { China } \\
\text { 2013-2016 }\end{array}$ & $\mathrm{H} \& \mathrm{~N}$ free flaps & 881 & 65 & Flap failure/necrosis (RR) \\
\hline $\mathrm{Yao}^{28}$ & Retrospective cohort & $\begin{array}{l}\text { University Health Network in } \\
\text { Toronto, Canada } \\
\text { 1997-2014 }\end{array}$ & $\begin{array}{l}\text { Free-flap reconstructions } \\
\text { for oral squamous cell } \\
\text { carcinoma }\end{array}$ & 365 & 39 & $\begin{array}{l}\text { Surgical site infection (RR), } \\
\text { plate exposure (RR) }\end{array}$ \\
\hline $\mathrm{Joo}^{29}$ & Retrospective cohort & $\begin{array}{l}\text { Catholic University of Korea, } \\
\text { Korea } \\
1993-2014 \\
\end{array}$ & $\begin{array}{l}\text { Free-flap reconstructions } \\
\text { for H\&N squamous cell } \\
\text { carcinoma }\end{array}$ & 259 & 40 & Surgical site infection (OR) \\
\hline Khan $^{30}$ & Retrospective cohort & $\begin{array}{l}\text { Mount Sinai Medical Center, } \\
\text { United States } \\
\text { 2007-2014 }\end{array}$ & $\mathrm{H} \& \mathrm{~N}$ free flaps & 415 & 46 & Surgical site infection (OR) \\
\hline \multicolumn{7}{|l|}{ Continued } \\
\hline
\end{tabular}




\begin{tabular}{|c|c|c|c|c|c|c|}
\hline References & Study Design & Database & Flap definition & No. of patients & No. of DM patients & Analyzed complication \\
\hline Brady $^{5}$ & Retrospective cohort & $\begin{array}{l}\text { National Surgical Quality } \\
\text { Improvement Program, } \\
\text { United States } \\
2005-2014\end{array}$ & $\mathrm{H} \& \mathrm{~N}$ free flaps & 2187 & 256 & $\begin{array}{l}\text { Total complications (RR), } \\
\text { surgical complications } \\
\text { (RR), return to OR (RR), } \\
\text { flap failure/necrosis (RR), } \\
\text { surgical site infection (RR), } \\
\text { dehiscence (RR), bleeding } \\
\text { (RR), other complications } \\
\text { (RR), readmission (RR), } \\
\text { mortality (RR) }\end{array}$ \\
\hline Bollig $^{31}$ & Retrospective cohort & $\begin{array}{l}\text { Missouri Hospital, United } \\
\text { States } \\
\text { 2009-2015 }\end{array}$ & $\mathrm{H} \& \mathrm{~N}$ free flaps & 203 & 91 & $\begin{array}{l}\text { Surgical complications (RR), } \\
\text { surgical site infection (RR), } \\
\text { venous thrombosis (RR) }\end{array}$ \\
\hline Eskander $^{32}$ & Retrospective cohort & $\begin{array}{l}\text { Ohio State University, United } \\
\text { States } \\
2006-2012\end{array}$ & $\mathrm{H} \& \mathrm{~N}$ free flaps & 515 & 84 & $\begin{array}{l}\text { Total complications (RR, } \\
\text { OR), surgical site infection } \\
\text { (RR, OR), dehiscence (RR), } \\
\text { other complications (RR) }\end{array}$ \\
\hline Rudolph $^{33}$ & Retrospective cohort & $\begin{array}{l}\text { Wake Forest Baptist Medical } \\
\text { Center, United States } \\
\text { 2008-2016 }\end{array}$ & $\begin{array}{l}\text { Cross-paramedian forehead } \\
\text { flaps for nasal reconstruc- } \\
\text { tions }\end{array}$ & 53 & 9 & Surgical complications (OR) \\
\hline Eskander ${ }^{35}$ & Retrospective cohort & $\begin{array}{l}\text { Ohio State University, United } \\
\text { States } \\
2006-2012\end{array}$ & $\mathrm{H} \& \mathrm{~N}$ free flaps & 515 & 84 & Readmission (RR) \\
\hline Crawley $^{36}$ & Retrospective cohort & $\begin{array}{l}\text { Thomas Jefferson University, } \\
\text { United States } \\
\text { 2006-2017 }\end{array}$ & $\mathrm{H} \& \mathrm{~N}$ free flaps & 889 & 128 & Flap failure/necrosis (RR) \\
\hline $\operatorname{Lin}^{37}$ & Retrospective cohort & $\begin{array}{l}\text { Kaohsiung Chang Gung } \\
\text { Memorial Hospital, Taiwan } \\
\text { 2008-2017 }\end{array}$ & $\begin{array}{l}\mathrm{H} \& \mathrm{~N} \text { free anterolateral thigh } \\
\text { flaps }\end{array}$ & 1284 & 251 & Surgical complications (RR) \\
\hline
\end{tabular}

Table 1. Patient Characteristics of the studies included in the meta-analysis $(n=27)$.

\section{Funnel Plot of Standard Error by Log odds ratio}

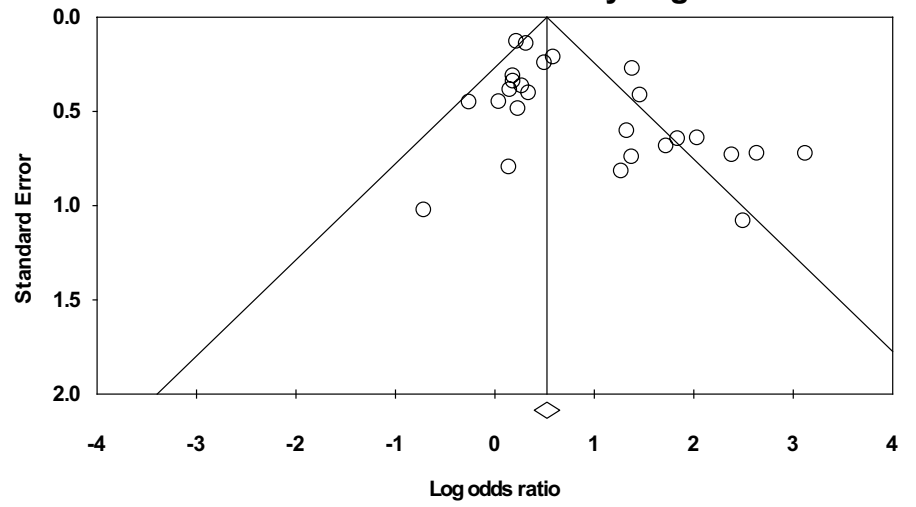

Figure 2. The funnel plot of all studies included.

Mortality. Two studies were included to evaluate mortality, with $291 \mathrm{DM}$ patients among 2610 subjects. All complication rates were expressed as RR. The pooled results showed that DM did not significantly increase mortality rates $(\mathrm{RR}=1.999,95 \% \mathrm{CI} 0.347-11.530, p=0.438)($ Fig. 6$)$.

Other complications. Two studies were included in the evaluation of other complications (e.g., myocardial infarction, cerebrovascular event, deep vein thrombosis, pulmonary embolism, pneumonia, urinary tract infection, and septic shock); a total of 2702 patients were enrolled, including $340 \mathrm{DM}$ patients. All complication rates were expressed as RR. The pooled results showed that DM significantly increased the risk of other complications $(\mathrm{RR}=1.284,95 \%$ CI 1.109-1.487, $p=0.001)$ (Fig. 6).

\section{Discussion}

DM has been investigated for potentially increasing the risk of postoperative complications, such as vascular occlusions and thrombosis, that jeopardize free flap survival and result in immune disturbances associated with poor wound healing ${ }^{8-10}$. However, retrospective cohort studies from different institutions and even different time periods within the same database could end up with contradicting conclusions $s^{4,5}$.

Previous meta-analyses exploring the association between DM and H\&N free-flap reconstructions had a few limitations. In 2015, Rosado et al. noted that DM was significantly related to more surgical complications following free-flap reconstructions in the $\mathrm{H} \& \mathrm{~N}$ region ${ }^{38}$. Consisting of merely five studies, this analysis seemed relatively short of conclusiveness ${ }^{38}$. Further investigations were performed by Cupato et al. ${ }^{39}$; these authors 
(a) Total complications

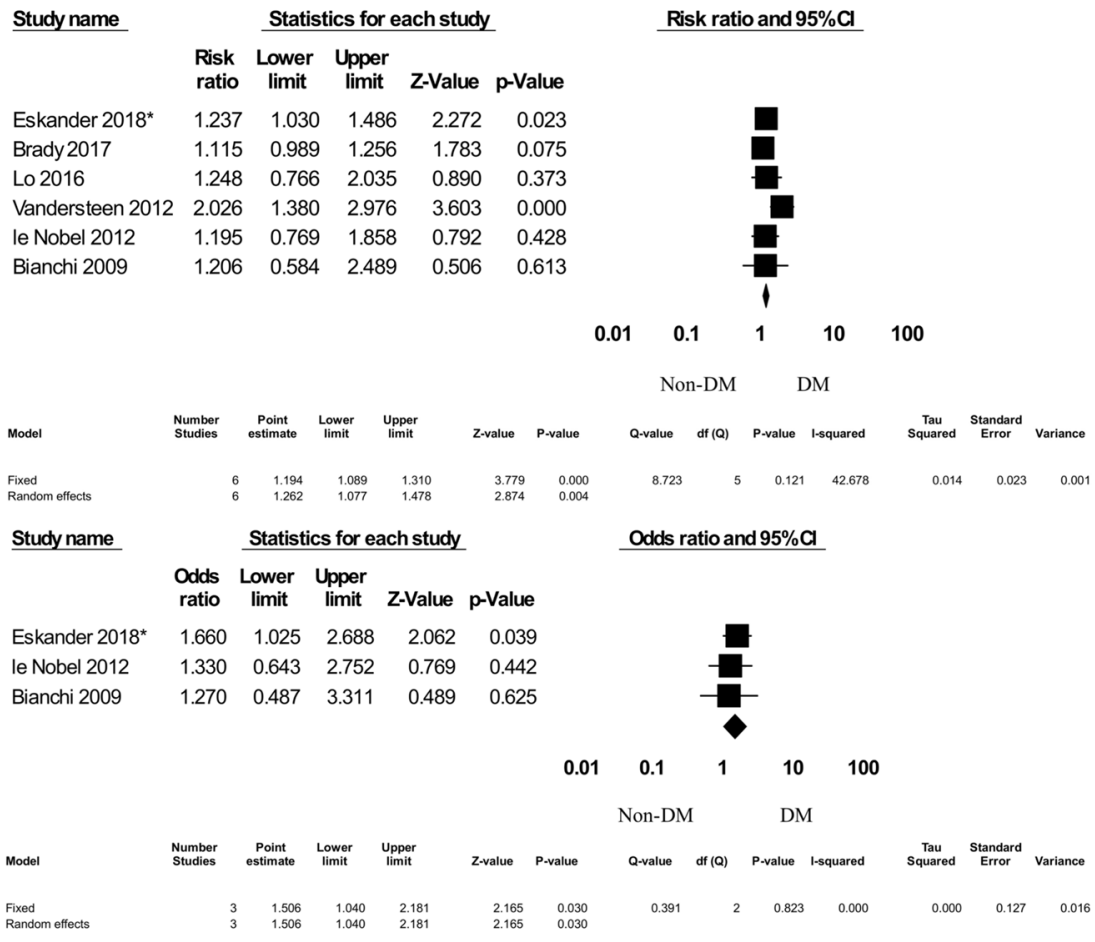

(b) Surgical complications

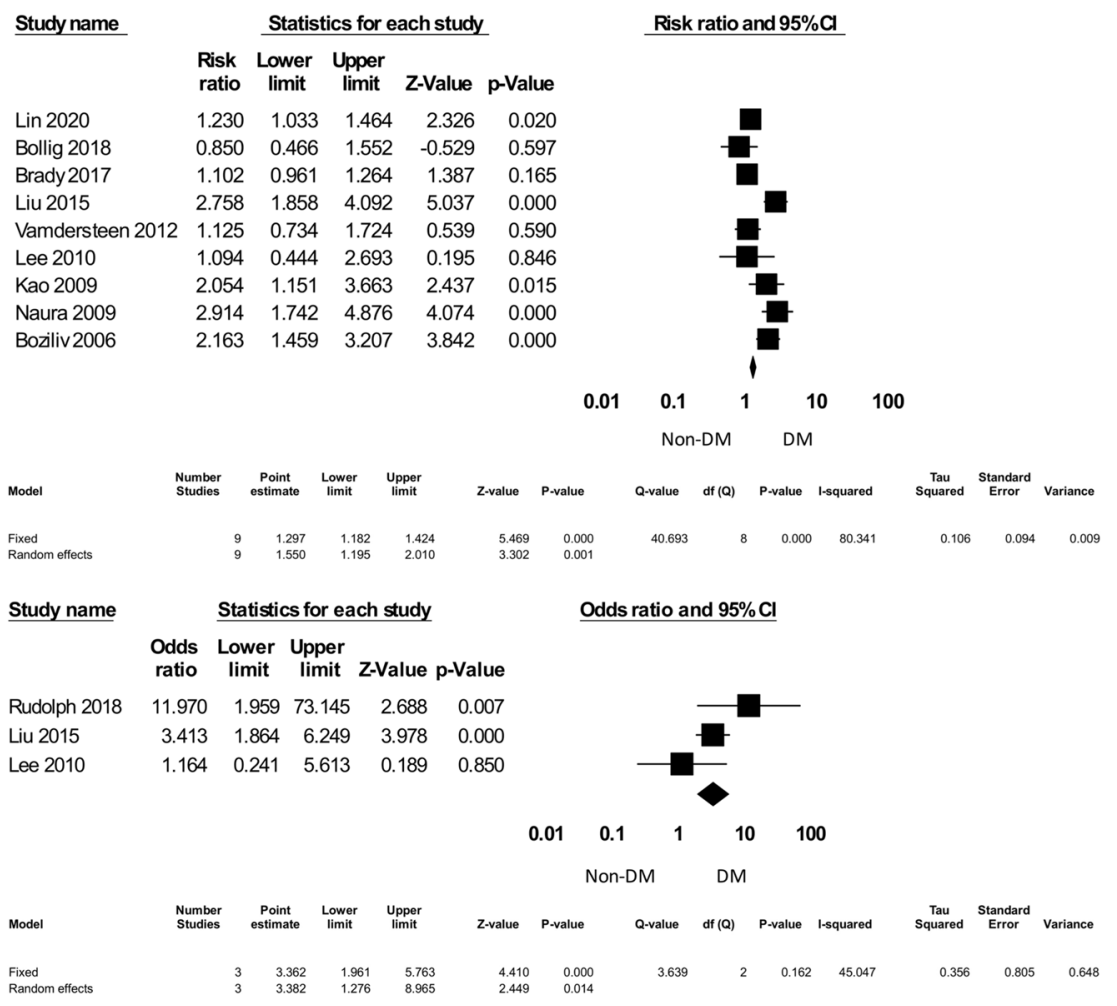

Figure 3. The postoperative complications forest plot: total and surgical complications. ${ }^{\star}$ Eskander $^{32}$. 
(a) Return to Operation Room (OR)

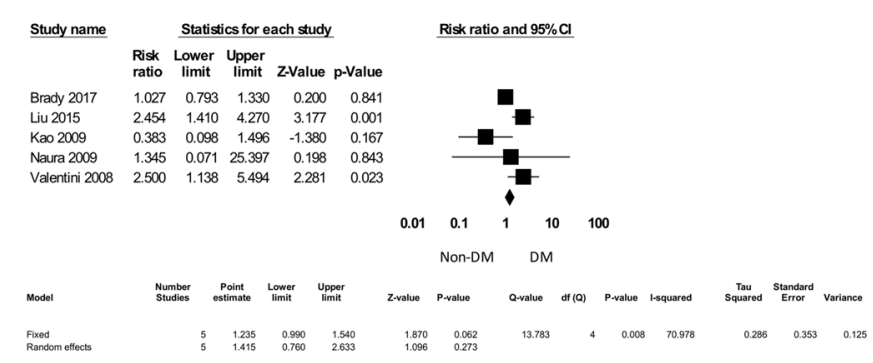

(b) Flap failure/necrosis

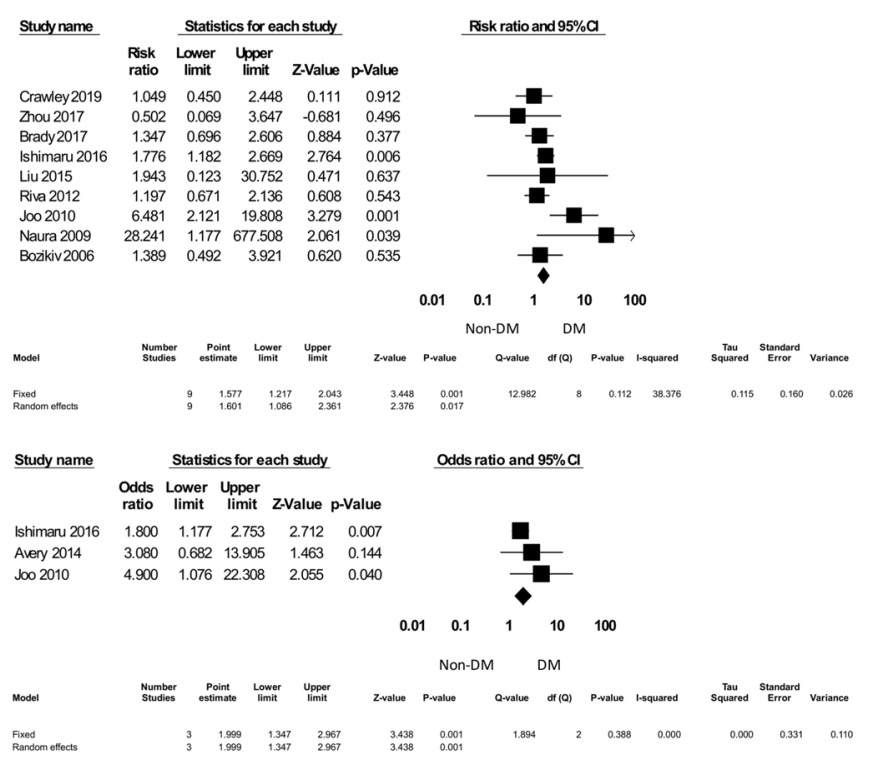

(c) Surgical Site Infection

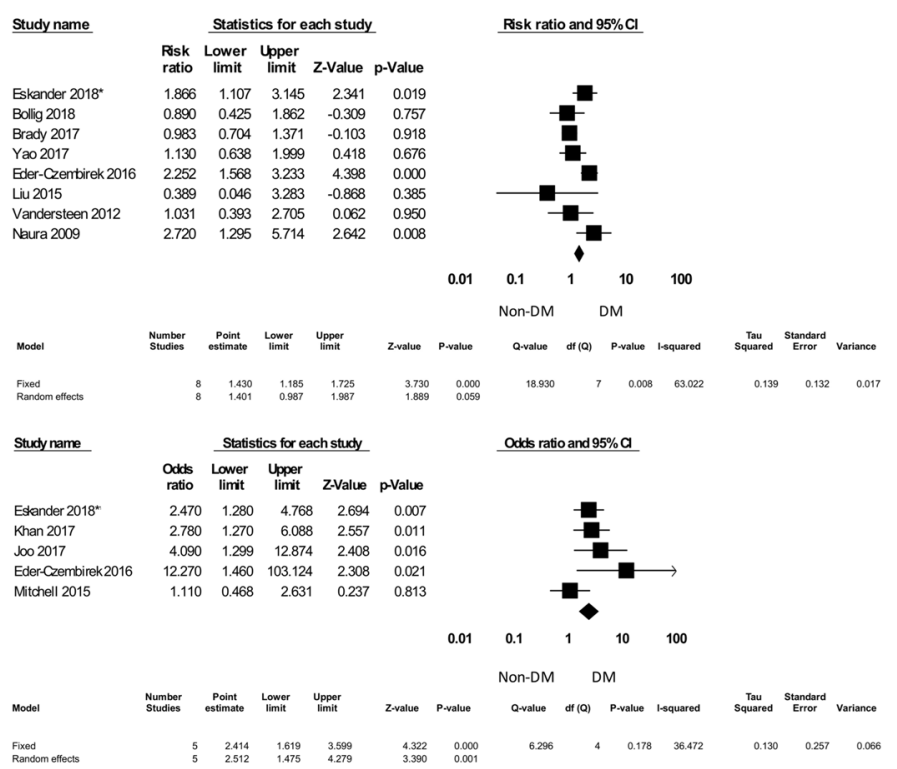

Figure 4. The individual surgical complications forest plot, part I: return to the operating room, flap failure/ necrosis, and surgical site infection. ${ }^{*}$ Eskander $^{32}$. 
(d) Dehiscence

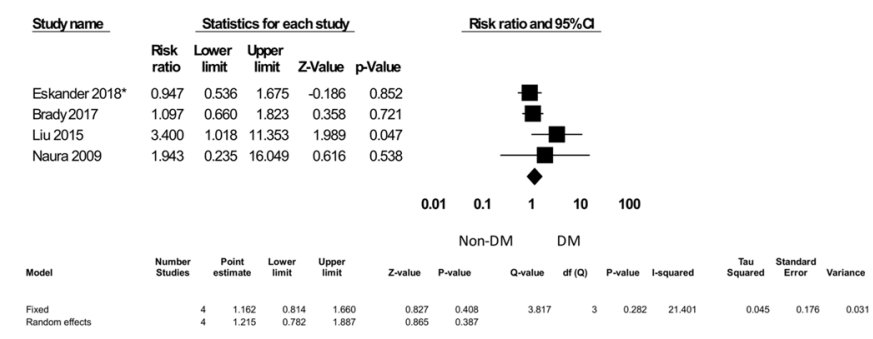

(e) Fistula

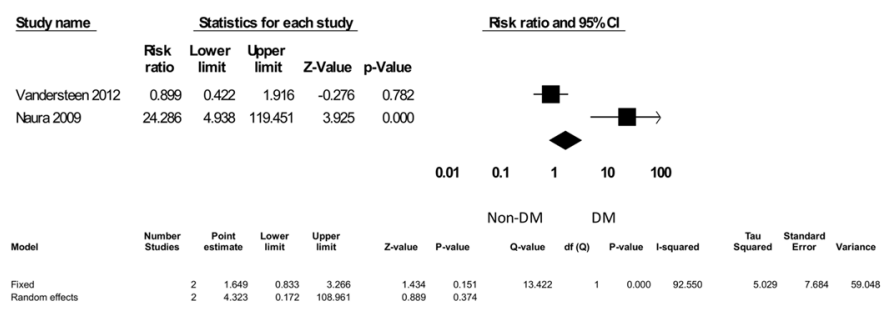

(f) Plate Exposure

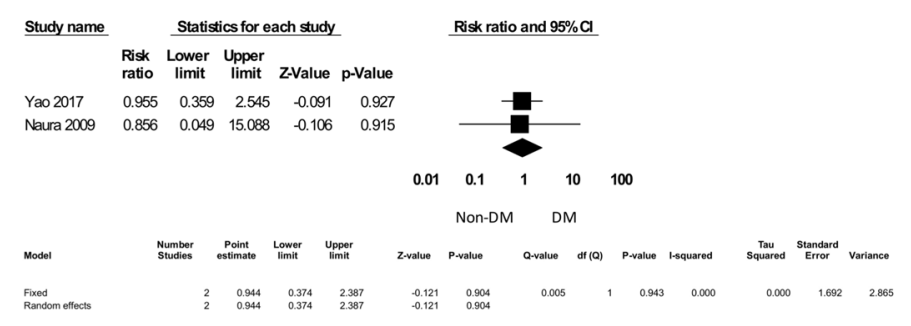

(g) Hematoma

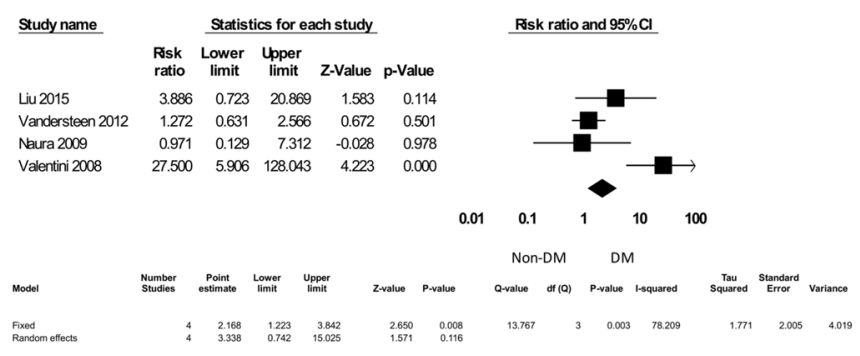

(h) Coagulation-related Complications (Hematoma, bleeding, requiring transfusion, thrombosis)

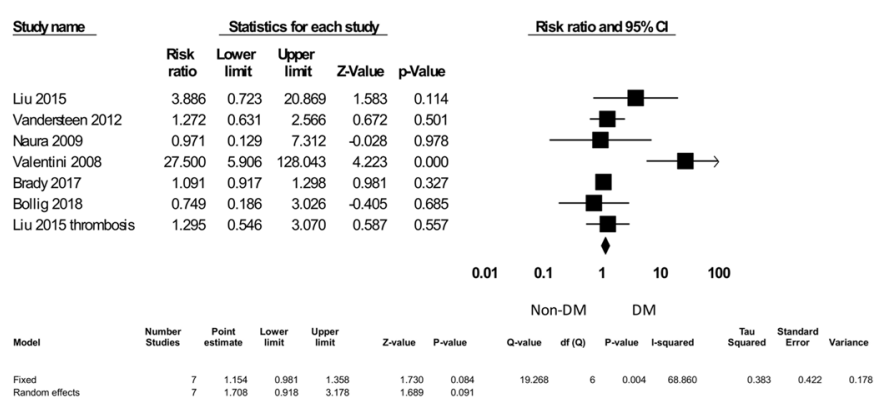

Figure 5. The individual surgical complications forest plot, part II: dehiscence, fistula, plate exposures, hematoma, and coagulation-related complications. ${ }^{\star} E_{\text {skander }}{ }^{32}$. 
(a) Re-admissions

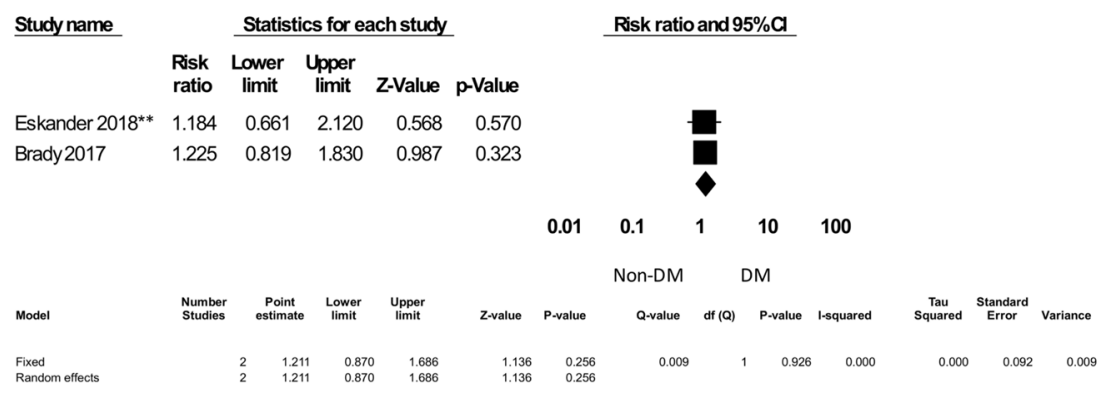

(b) Mortality

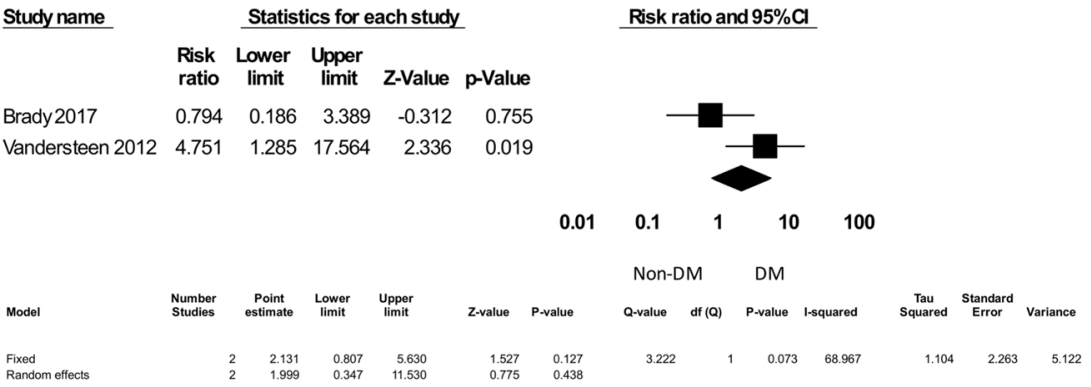

(c) Other Complications
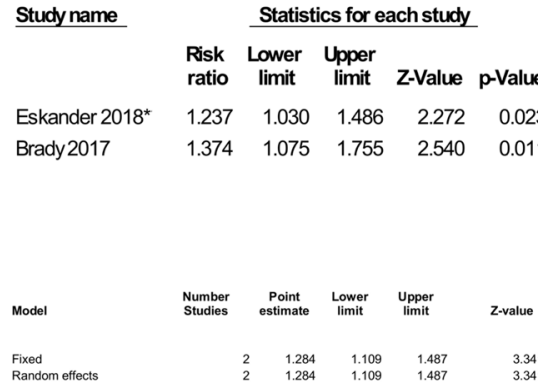

Risk ratio and $95 \% \mathrm{Cl}$

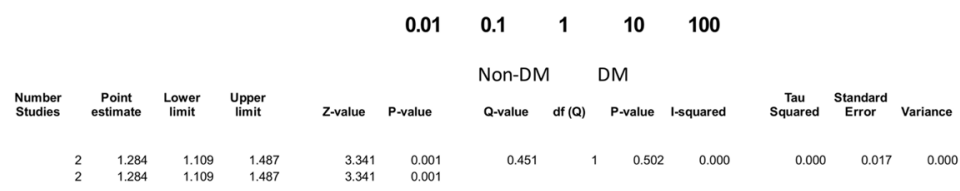

Figure 6. Quality aspects: readmissions, mortality, and other complications. ${ }^{\star}$ Eskander $^{32},{ }^{*} \operatorname{Eskander}^{35}$. Other complications include myocardial infarction, cerebrovascular event, deep vein thrombosis, pulmonary embolism, pneumonia, urinary tract infection, and septic shock.

gathered 16 studies for meta-analysis. They concluded that DM significantly increased flap failures and local region complications ${ }^{39}$. This complication analysis was performed to include all major H\&N surgeries instead of limiting the analysis to free-flap reconstructions ${ }^{39}$. They also pooled different complications under the term "locoregional" 39 . None of these meta-analyses was explicitly designed to analyze individual complications. Additionally, neither one considered crucial complications nor quality indicators in their studies. For surgeons, a better understanding of which complication was at higher risk might be more helpful and provide insights into routine or pragmatic formulations in clinical practice.

In this study, a total of 27 studies regarding $H \& N$ free-flap reconstructions in patients with DM were included in a meta-analysis in response to preceding studies with different opinions about free-flap failure or abnormal healing of the anastomoses in DM subjects ${ }^{5,12,40,41}$. Some studies concluded that patients with DMS were at increased risk and others did not ${ }^{5,12,40,41}$. Overall, we found that DM was associated with increasing total complications (surgical and other clinical sequelae), return to the operating room, and free-flap failure after the operation (Figs. 3 and 4). The underlying mechanisms might be due to the high prevalence of peripheral vascular diseases in DM patients ${ }^{8}$. Peripheral vascular diseases can result in fragile vessel conditions and precipitate free-flap failure. Currently, vascular mapping for evaluating vascular viability and anatomy by color Doppler, computed tomography (CT) angiography or magnetic resonance imaging (MRI) angiography might improve microsurgical outcomes ${ }^{42}$. Therefore, adopting angiography as part of the preoperative assessments might be beneficial and suggested for routine used in DM patients referred for free-flap operations.

$\mathrm{DM}$ has been demonstrated to alter the immune system with cytokine effects on local wound healing ${ }^{10}$. Higher infection rates in DM patients have been previously reported from various types of surgical procedures ${ }^{6}$. In our 
study, surgical site infection after free-flap reconstruction was significantly increased in DM patients $(\mathrm{RR}=1.401$, $p=0.059 ; \mathrm{OR}=2.414, p<0.001$ ) (Fig. 4). Therefore, hyperglycemia should be taken seriously and controlled cautiously to reduce surgical site infections in DM patients before and after surgery.

The ideal target for glycemic control before and after surgery is still a debated issue. In some studies, maintaining $\mathrm{HbAlc}$ less than $8.5 \%$ (69 $\mathrm{mmol} / \mathrm{mol})$ for the general population undergoing surgeries is highly suggested ${ }^{43}$. However, there is no consensus about whether DM patients planning to undergo free-flap reconstructions should maintain stricter glycemic control. Currently, intensive blood sugar control of less than $150 \mathrm{mg} / \mathrm{dL}$ before and after surgery might reduce the risk of surgical site infection ${ }^{44}$. Both the Society of Thoracic Surgeons (STS) and the American Association of Clinical Endocrinologists and American Diabetes Association (AACE/ADA) endorse a glycemic range between 140 and $180 \mathrm{mg} / \mathrm{dL}$ for postoperative patients ${ }^{45}$.

A series of analyses according to the infection-related complications were further performed. Interestingly, DM was not associated with dehiscence, fistulas, or plate exposure (Fig. 5). Early empirical antibiotic usage or switching, surgical intervention and return to the operating room might control infection and deter such serious and long-term complications. However, there has been no consensus regarding infection prophylaxis and management in patients with DM following microsurgical reconstructions ${ }^{23,46}$.

Pharmacologic prophylaxis with antiplatelets, anticoagulants, and volume expanders has been commonly prescribed after free flap transfers in DM patients due to their impaired microcirculation ${ }^{47}$. In this study, of the risk of hematoma was not increasing in diabetic flap recipients (Fig. 5). However, there was a trend of increasing total coagulation-related complications (e.g., hematoma, bleeding, required transfusion, and thrombosis) in the DM group ( $R R=1.708,95 \%$ CI $0.918-3.178, p=0.091)$ (Fig. 5). Therefore, surgeons should be cautious in dose adjustment and coagulation monitoring while using antiplatelets or anticoagulants in DM patients after surgery ${ }^{47}$.

Regarding the quality aspects, we found that DM did not significantly increase readmission rates or mortality after free-flap reconstruction. However, other common clinical complications, including myocardial infarction, cerebrovascular event, deep vein thrombosis, pulmonary embolism, pneumonia, urinary tract infection, and septic shock, were noted to be increased after surgery in DM patients. It might be that those patients with H\&N malignancies seemed to suffer from more comorbidities than the general population ${ }^{48,49}$. For instance, the high prevalence of tobacco and alcohol use in those patients imposes higher cardiovascular diseases, which could be exacerbated during surgery ${ }^{50,51}$. Additionally, blood transfusion during or after surgery might also aggravate heart failure, respiratory distress, and pneumonia ${ }^{52}$. Aside from cancer recurrence or metastasis, the most common causes of mortality after free-flap reconstruction were cardiac, pulmonary, and infectious etiologies ${ }^{53}$. Therefore, pre- or postoperative care should be more attentive for DM patients undergoing H\&N reconstructions. A thorough examination of cardiopulmonary function and comprehensive comorbidity treatments should be implemented before the operation. In addition to surgical teams, different specialties might be consulted beforehand to avoid serious medical consequences.

\section{Conclusion}

In summary, DM patients are prone to develop various complications after $\mathrm{H} \& \mathrm{~N}$ free-flap reconstructions, and more aggressive strategies should be taken to ensure better outcomes. Our study results suggest practical ways for surgeons and oncologists to evaluate the risk of surgery in these patients. A patient-based and individual decision-making process should always be implemented and cautiously reviewed before free-flap reconstruction.

Received: 6 November 2020; Accepted: 1 March 2021

Published online: 16 March 2021

\section{References}

1. Wong, C. H. \& Wei, F. C. Microsurgical free flap in head and neck reconstruction. Head Neck 32, 1236-1245. https://doi. org/10.1002/hed.21284 (2010).

2. Richardson, A. E., Broadbent, E. \& Morton, R. P. A systematic review of psychological interventions for patients with head and neck cancer. Support Care Cancer 27, 2007-2021. https://doi.org/10.1007/s00520-019-04768-3 (2019).

3. Pattani, K. M., Byrne, P., Boahene, K. \& Richmon, J. What makes a good flap go bad? A critical analysis of the literature of intraoperative factors related to free-flap failure. Laryngoscope 120, 717-723. https://doi.org/10.1002/lary.20825 (2010).

4. Kantar, R. S. et al. Diabetes is not associated with increased rates of free-flap failure: analysis of outcomes in 6030 patients from the ACS-NSQIP database. Microsurgery 39, 14-23. https://doi.org/10.1002/micr.30332 (2019).

5. Brady, J. S. et al. Impact of diabetes on free flap surgery of the head and neck: a NSQIP analysis. Microsurgery 38, 504-511. https ://doi.org/10.1002/micr.30276 (2018).

6. Martin, E. T. et al. Diabetes and risk of surgical site infection: a systematic review and meta-analysis. Infect. Control Hosp. Epidemiol. 37, 88-99. https://doi.org/10.1017/ice.2015.249 (2016).

7. Ogihara, H., Takeuchi, K. \& Majima, Y. Risk factors of postoperative infection in head and neck surgery. Auris Nasus Larynx 36, 457-460. https://doi.org/10.1016/j.anl.2008.10.005 (2009).

8. Paneni, F., Beckman, J. A., Creager, M. A. \& Cosentino, F. Diabetes and vascular disease: pathophysiology, clinical consequences, and medical therapy: part I. Eur. Heart J. 34, 2436-2443. https://doi.org/10.1093/eurheartj/eht149 (2013).

9. Vazzana, N., Ranalli, P., Cuccurullo, C. \& Davi, G. Diabetes mellitus and thrombosis. Thromb. Res. 129, 371-377. https://doi. org/10.1016/j.thromres.2011.11.052 (2012).

10. Brem, H. \& Tomic-Canic, M. Cellular and molecular basis of wound healing in diabetes. J. Clin. Investig. 117, 1219-1222. https:// doi.org/10.1172/jci32169 (2007).

11. Bozikov, K. \& Arnez, Z. M. Factors predicting free flap complications in head and neck reconstruction. J. Plast. Reconstr. Aesthet. Surg. 59, 737-742. https://doi.org/10.1016/j.bjps.2005.11.013 (2006).

12. Valentini, V. et al. Diabetes as main risk factor in head and neck reconstructive surgery with free flaps. J. Craniofac. Surg. 19, 1080-1084. https://doi.org/10.1097/SCS.0b013e3181763531 (2008).

13. Nuara, M. J., Sauder, C. L. \& Alam, D. S. Prospective analysis of outcomes and complications of 300 consecutive microvascular reconstructions. Arch. Facial Plast. Surg. 11, 235-239. https://doi.org/10.1001/archfacial.2009.46 (2009). 
14. Kao, H. K. et al. The impacts of liver cirrhosis on head and neck cancer patients undergoing microsurgical free tissue transfer: an evaluation of flap outcome and flap-related complications. Oral Oncol. 45, 1058-1062. https://doi.org/10.1016/j.oraloncolo gy.2009.07.010 (2009).

15. Bianchi, B., Copelli, C., Ferrari, S., Ferri, A. \& Sesenna, E. Free flaps: outcomes and complications in head and neck reconstructions. J. Craniomaxillofac. Surg. 37, 438-442. https://doi.org/10.1016/j.jcms.2009.05.003 (2009).

16. Joo, Y. H., Sun, D. I., Park, J. O., Cho, K. J. \& Kim, M. S. Risk factors of free flap compromise in 247 cases of microvascular head and neck reconstruction: a single surgeon's experience. Eur. Arch. Otorhinolaryngol. 267, 1629-1633. https://doi.org/10.1007/s0040 5-010-1268-1 (2010).

17. Lee, S. \& Thiele, C. Factors associated with free flap complications after head and neck reconstruction and the molecular basis of fibrotic tissue rearrangement in preirradiated soft tissue. J. Oral Maxillofac. Surg. 68, 2169-2178. https://doi.org/10.1016/j. joms.2009.08.026 (2010).

18. le Nobel, G. J., Higgins, K. M. \& Enepekides, D. J. Predictors of complications of free flap reconstruction in head and neck surgery: analysis of 304 free flap reconstruction procedures. Laryngoscope 122, 1014-1019. https://doi.org/10.1002/lary.22454 (2012).

19. Vandersteen, C. et al. Impact of patient comorbidities on head and neck microvascular reconstruction. A report on 423 cases. Eur. Arch. Otorhinolaryngol. 270, 1741-1746. https://doi.org/10.1007/s00405-012-2224-z (2013).

20. Riva, F. M. et al. The outcome of prostaglandin-E1 and dextran-40 compared to no antithrombotic therapy in head and neck free tissue transfer: analysis of 1,351 cases in a single center. Microsurgery 32, 339-343. https://doi.org/10.1002/micr.21958 (2012).

21. Avery, C. M., Gandhi, N., Peel, D. \& Neal, C. P. Indications and outcomes for 100 patients managed with a pectoralis major flap within a UK maxillofacial unit. Int. J. Oral Maxillofac. Surg. 43, 546-554. https://doi.org/10.1016/j.ijom.2013.10.009 (2014).

22. Liu, Z. et al. Microvascular reconstruction in elderly oral cancer patients: Does diabetes status have a predictive role in free flap complications?. J. Oral Maxillofac. Surg. 73, 357-369. https://doi.org/10.1016/j.joms.2014.08.009 (2015).

23. Mitchell, R. M., Mendez, E., Schmitt, N. C., Bhrany, A. D. \& Futran, N. D. Antibiotic prophylaxis in patients undergoing head and neck free flap reconstruction. JAMA Otolaryngol. Head Neck Surg. 141, 1096-1103. https://doi.org/10.1001/jamaoto.2015.0513 (2015).

24. Lo, S. L., Yen, Y. H., Lee, P. J., Liu, C. C. \& Pu, C. M. Factors influencing postoperative complications in reconstructive microsurgery for head and neck cancer. J. Oral Maxillofac. Surg. 75, 867-873. https://doi.org/10.1016/j.joms.2016.09.025 (2017).

25. Ishimaru, M. et al. Risk factors for free-flap failure in 2,846 patients with head and neck cancer: a national database study in Japan. J. Oral Maxillofac. Surg. 74, 1265-1270. https://doi.org/10.1016/j.joms.2016.01.009 (2016).

26. Eder-Czembirek, C., Czembirek, C., Braun, P., Perisanidis, C. \& Seemann, R. Surgical site infection in clean-contaminated wounds after multimodal treatment of advanced oral squamous cell carcinoma. J. Craniomaxillofac. Surg. 44, 1957-1962. https://doi. org/10.1016/j.jcms.2016.09.013 (2016).

27. Zhou, W. et al. Risk factors for free-flap failure: a retrospective analysis of 881 free flaps for head and neck defect reconstruction. Int. J. Oral Maxillofac. Surg. 46, 941-945. https://doi.org/10.1016/j.ijom.2017.03.023 (2017).

28. Yao, C. M. et al. Surgical site infections following oral cavity cancer resection and reconstruction is a risk factor for plate exposure. J. Otolaryngol. Head Neck Surg. 46, 30. https://doi.org/10.1186/s40463-017-0206-2 (2017).

29. Joo, Y. H., Cho, K. J., Park, J. O., Kim, S. Y. \& Kim, M. S. Surgical morbidity and mortality in patients after microvascular reconstruction for head and neck cancer. Clin. Otolaryngol. 43, 502-508. https://doi.org/10.1111/coa.13006 (2018).

30. Khan, M. N. et al. Association of body mass index with infectious complications in free tissue transfer for head and neck reconstructive surgery. JAMA Otolaryngol. Head Neck Surg. 143, 574-579. https://doi.org/10.1001/jamaoto.2016.4304 (2017).

31. Bollig, C. A., Spradling, C. S., Dooley, L. M., Galloway, T. L. \& Jorgensen, J. B. Impact of perioperative hyperglycemia in patients undergoing microvascular reconstruction. Head Neck 40, 1196-1206. https://doi.org/10.1002/hed.25097 (2018).

32. Eskander, A. et al. Predictors of complications in patients receiving head and neck free flap reconstructive procedures. Otolaryngol. Head Neck Surg. 158, 839-847. https://doi.org/10.1177/0194599818757949 (2018).

33. Rudolph, M. A., Walker, N. J., Rebowe, R. E. \& Marks, M. W. Broadening applications and insights into the cross-paramedian forehead flap over a 19-year period. J. Plast. Reconstr. Aesthet. Surg. 72, 763-770. https://doi.org/10.1016/j.bjps.2018.12.001 (2019).

34. Offodile, A. C. 2nd. et al. Hyperglycemia and risk of adverse outcomes following microvascular reconstruction of oncologic head and neck defects. Oral Oncol. 79, 15-19. https://doi.org/10.1016/j.oraloncology.2018.02.009 (2018).

35. Eskander, A. et al. Quality indicators: measurement and predictors in head and neck cancer free flap patients. Otolaryngol. Head Neck Surg. 158, 265-272. https://doi.org/10.1177/0194599817742373 (2018).

36. Crawley, M. B. et al. Factors associated with free-flap failures in head and neck reconstruction. Otolaryngol. Head Neck Surg. 161, 598-604. https://doi.org/10.1177/0194599819860809 (2019).

37. Lin, P. C., Kuo, P. J., Kuo, S. C. H., Chien, P. C. \& Hsieh, C. H. Risk factors associated with postoperative complications of free anterolateral thigh flap placement in patients with head and neck cancer: analysis of propensity score-matched cohorts. Microsurgery 40, 538-544. https://doi.org/10.1002/micr.30587 (2020).

38. Rosado, P., Cheng, H. T., Wu, C. M. \& Wei, F. C. Influence of diabetes mellitus on postoperative complications and failure in head and neck free flap reconstruction: a systematic review and meta-analysis. Head Neck 37, 615-618. https://doi.org/10.1002/hed.23624 (2015).

39. Caputo, M. P. et al. Diabetes mellitus in major head and neck cancer surgery: systematic review and meta-analysis. Head Neck https://doi.org/10.1002/hed.26349 (2020).

40. Nahabedian, M. Y., Momen, B. \& Manson, P. N. Factors associated with anastomotic failure after microvascular reconstruction of the breast. Plast. Reconstr. Surg. 114, 74-82. https://doi.org/10.1097/01.prs.0000127798.69644.65 (2004).

41. Cooley, B. C., Hanel, D. P., Anderson, R. B., Foster, M. D. \& Gould, J. S. The influence of diabetes on free flap transfer: I. Flap survival and microvascular healing. Ann. Plast. Surg. 29, 58-64. https://doi.org/10.1097/00000637-199207000-00012 (1992).

42. Pratt, G. F. et al. Preoperative imaging for perforator flaps in reconstructive surgery: a systematic review of the evidence for current techniques. Ann. Plast. Surg. 69, 3-9. https://doi.org/10.1097/SPA.0b013e318222b7b7 (2012).

43. Dhatariya, K. How to prepare people with diabetes for surgery. Diabetes Primary Care 20, 53-54 (2018).

44. de Vries, F. E. et al. Meta-analysis of lower perioperative blood glucose target levels for reduction of surgical-site infection. $\mathrm{Br}$. J. Surg. 104, e95-e105. https://doi.org/10.1002/bjs.10424 (2017).

45. Sudhakaran, S. \& Surani, S. R. Guidelines for perioperative management of the diabetic patient. Surg. Res. Pract. 2015, 284063. https://doi.org/10.1155/2015/284063 (2015).

46. Carroll, W. R. et al. Three-dose vs extended-course clindamycin prophylaxis for free-flap reconstruction of the head and neck. Arch. Otolaryngol. Head Neck Surg. 129, 771-774. https://doi.org/10.1001/archotol.129.7.771 (2003).

47. McMillan, D. E. Deterioration of the microcirculation in diabetes. Diabetes 24, 944-957. https://doi.org/10.2337/diab.24.10.944 (1975).

48. Landis, S. H. et al. Prevalence and incidence of acute and chronic comorbidity in patients with squamous cell carcinoma of the head and neck. Head Neck 34, 238-244. https://doi.org/10.1002/hed.21720 (2012).

49. Piccirillo, J. F. \& Vlahiotis, A. Comorbidity in patients with cancer of the head and neck: prevalence and impact on treatment and prognosis. Curr Oncol Rep 8, 123-129. https://doi.org/10.1007/s11912-006-0047-z (2006).

50. Leon, B. M. \& Maddox, T. M. Diabetes and cardiovascular disease: epidemiology, biological mechanisms, treatment recommendations and future research. World J. Diabetes 6, 1246-1258. https://doi.org/10.4239/wjd.v6.i13.1246 (2015). 
51. Datema, F. R., Poldermans, D. \& Baatenburg de Jong, R. J. Incidence and prediction of major cardiovascular complications in head and neck surgery. Head Neck 32, 1485-1493. https://doi.org/10.1002/hed.21351 (2010).

52. Puram, S. V. et al. Transfusion in head and neck free flap patients: practice patterns and a comparative analysis by flap type. Otolaryngol. Head Neck Surg. 152, 449-457. https://doi.org/10.1177/0194599814567107 (2015).

53. Pohlenz, P., Klatt, J., Schmelzle, R. \& Li, L. The importance of in-hospital mortality for patients requiring free tissue transfer for head and neck oncology. Br. J. Oral Maxillofac. Surg. 51, 508-513. https://doi.org/10.1016/j.bjoms.2012.10.020 (2013).

\section{Acknowledgments}

The authors appreciated Wen-Pin Lien MD. PhD. for his valuable revision of this manuscript.

\section{Author contributions}

T.-H.C.: Collected, analyzed the data and worked for the drafting of the manuscript. C.-K.T.: Critically reviewed the manuscript for important intellectual content. C.-T.T.: Critically reviewed the manuscript for important intellectual content. J.-W.L.: Critically reviewed the manuscript for important intellectual content. F.-T.C.: Critically reviewed the manuscript for important intellectual content. S.-N.C.: Designed the whole study, analyzed and interpreted the data; Critically reviewed the manuscript for important intellectual content. J.-J.H.: Designed the whole study, analyzed and interpreted the data.

\section{Funding}

None.

\section{Competing interests}

The authors declare no competing interests.

\section{Additional information}

Correspondence and requests for materials should be addressed to S.-N.C. or J.-J.H.

Reprints and permissions information is available at www.nature.com/reprints.

Publisher's note Springer Nature remains neutral with regard to jurisdictional claims in published maps and institutional affiliations.

(c) (i) Open Access This article is licensed under a Creative Commons Attribution 4.0 International License, which permits use, sharing, adaptation, distribution and reproduction in any medium or format, as long as you give appropriate credit to the original author(s) and the source, provide a link to the Creative Commons licence, and indicate if changes were made. The images or other third party material in this article are included in the article's Creative Commons licence, unless indicated otherwise in a credit line to the material. If material is not included in the article's Creative Commons licence and your intended use is not permitted by statutory regulation or exceeds the permitted use, you will need to obtain permission directly from the copyright holder. To view a copy of this licence, visit http://creativecommons.org/licenses/by/4.0/.

(C) The Author(s) 2021 